\title{
Comparison of Patients with Rheumatoid Arthritis in Disease Remission in the European Union and United States
}

\author{
Siva Narayanan, MS MHS \\ SVP and Global Head, Global Evidence, Value and Access \\ Ipsos Healthcare, $2020 \mathrm{~K}$ Street, Washington, DC, 20006, USA \\ Phone: +1 301-605-3663 \\ Email: siva_narayanan[at]verizon.net
}

\begin{abstract}
To compare patients with Rheumatoid Arthritis (RA) on disease remission in the European Union (EU) and United States (US) among those receiving a biologic treatment as part of usual care, a multi-country medical chart-review study was conducted in 2011 among patients recently treated with a biologic as part of usual care. Analysis included 1161 and 405 RA patients experiencing disease remission in the $\mathrm{EU}$ and $\mathrm{US}$ respectively; mean duration in remission was: EU/US:12.2mo/12.1mo. Among those with lab measures, Rheumatoid Factor and Anti-CCP results were similar, and so as the disease severity scores (e.g., Swollen Joint Count, $100 \mathrm{~mm}$ VAS score, HAQ and DAS28). CRP values, Tender Joint Count and Total Sharp Scores were higher among the EU cohort. RA patients in remission were found mostly similar between these geographic clusters, despite the potential variations in healthcare systems and modalities of care delivery, possibly attributed to EULAR/ACR efforts in standardizing the Treat-to-Target guidelines, with a focus on clinical remission.
\end{abstract}

Keywords: Rheumatoid Arthritis, Remission, Biologics, Europe, US

\section{Introduction}

Rheumatoid Arthritis (RA) is the most common chronic autoimmune disease that results in systemic inflammation and primarily attacks synovial joints $[1,2]$. RA is associated with significant mortality and morbidity, including diminished health related quality of life, cognitive dysfunction and work disability [3-5].

The treatment paradigm for RA has changed dramatically over the last two decades, with more effective interventions introduced to prevent joint damage and functional impairment. Treatments for RA rest on use of disease-modifying antirheumatic drugs (DMARDS) including conventional synthetic DMARDs, biological DMARDS and targeted synthetic DMARDS [6-8].The potent therapeutic interventions enable targeting specific components of the immune system, which in turn allows efficient suppression of the pathologic inflammation cascade which causes joint destruction. On the other hand, treatment with biologics leaves the patient more susceptible to infection by inducing a certain extent of immuno-suppression [9].

European League Against Rheumatism (EULAR) and the American College Rheumatology (ACR) have evidencedriven recommendations for the use of pharmacotherapies, weighing the risk-benefits of the pharmacotherapies; a key recommendation that consistently found its place in the EULAR/ACR guidelines in the recent years is to initiate appropriate therapy soon after diagnosis in order to achieve clinical remission (preferred), or low disease activity $[6-8,10,11]$. As clinical practices in the US and Europe adopt the EULAR/ACR guidelines/recommendations, the extent to which patients who were managed towards remission were in fact maintained in remission and what their clinical characteristics were in the respective geographies is unknown. This study objective was to address this evidence gap in portraying the characteristics of RA patients in Europe and the United States (US) in disease remission, per physician's clinical assessment/judgment.

\section{Material and Methods}

The study was a multi-country, multi-center retrospective medical chart review of adult (16-89 years old) RA patients conducted among physicians in the US and big-5 European countries (5EU), namely, the United Kingdom (UK), France, Germany, Italy and Spain. Physicians (mainly, rheumatologists)were sampled in each of the countries using online physician panels to attain a geographically representative sample in the respective countries. Invitations to participate in the research were sent to a random set of physicians in the existing online physician panels. The physicians representing both hospital-based and private practices in each country, personally responsible for choosing and prescribing treatment for patients with RA, and treating a minimum of 2RA patients per week and having 3-30 years of clinical practice experience were screened for study participation. Each physician reported de-identified anonymous data on patients who were recently treated with a biologic as part of usual care. Up to 5study-eligible RA patient charts were randomly selected by each physician from a sample of prospective patients visiting their respective center/practice during the study screening period in OctDec 2011.

The electronic data collection form was used to collect the following data elements from eligible patient charts: patient demographics, laboratory values, treatment patterns/dynamics andppatient symptomatology/disease status. Erythrocyte sedimentation rate $(\mathrm{ESR}, \mathrm{mm} / \mathrm{h}), \mathrm{C}$ - 


\section{International Journal of Science and Research (IJSR) ISSN (Online): 2319-7064}

Index Copernicus Value (2013): 6.14 | Impact Factor (2015): 6.391

reactive protein $(\mathrm{CRP}, \mathrm{mg} / \mathrm{dl})$, Rheumatoid Factor, anticyclic citrullinated peptide antibody (Anti-CCP), and human leukocyte antigen B27 (HLA-B27) measures as well as Tender Joint Count (TJC), Swollen Joint Count (SJC), 100mm Visual Analog Scale(VAS), Health Assessment Questionnaire (HAQ), Disease Activity Score in 28 joints (DAS28)and Total Sharp Score as documented in patient charts were abstracted. Physicians assessed patient disease status per clinical judgment (both objective and subjective) to indicate whether the patient was in "disease remission" at the time of chart abstraction.

Only de-identified anonymous data was collected from the patient charts by the treating physicians. This mode of data collection method met the criteria for local ethics review exemption per the respective physician/site requirements in the respective countries. RA patients currently experiencing disease remission were included in this analysis. Descriptive statistics were utilized to analyze the data, comparing the patients currently in remission in the 5EU and the US. Statistical differences were assessed using chi-square tests for categorical variables or t-tests for continuous variables; p-values of $<0.05$ were considered significant in all analyses.

\section{Results}

Overall, data corresponding to 1566 eligible RA patients (5EU: 1161, US: 405) who were in disease remission (per physician assessment) at the time of the study were included in the study. Mean age of these RA patients were 51.6 years and 51.9 years in the 5EU and US respectively; $70.1 \%$ and $72.3 \%$ were female in 5EU and US respectively. At the time of the study, the average duration of remission for RA patients in 5EU and US was 12.2 months (range: 7.5 months (Italy) - 14.8 months (UK))) and 12.1 months respectively.

Majority of these patients, specifically, $75 \%$ in $5 \mathrm{EU}$ and $74 \%$ in the US were on their 1 st line biologics, while $20 \%$ and $22 \%$ were respectively on $2^{\text {nd }}$ line biologics in EU5 and the US [Table 1].The average duration of remission was similar among the patients on $1^{\text {st }}$ line biologics (5EU: 12.3 months (range: 7.3 months (Italy) - 15.0 (France))); US: 12.7 months); patients on $2^{\text {nd }}$ line biologics in the $5 \mathrm{EU}$ on average had a slightly longer duration of remission (12.1 months; range: 8.6 months (Italy) - 16.3 months $(\mathrm{UK}))$ in comparison to their US counterparts (11.3 months).

Table 1: Patient's Current Treatment Line

\begin{tabular}{|c|c|c|}
\hline \multirow{2}{*}{} & \multicolumn{2}{|c|}{ RA Patients in Disease Remission } \\
\cline { 2 - 3 } & $5 \mathrm{EU}(\mathrm{N}=1161)$ & $\mathrm{US}(\mathrm{N}=405)$ \\
\hline $\begin{array}{c}\text { Patients on } 1^{\text {st }} \text { line RA } \\
\text { biologic treatment, \% }\end{array}$ & $75 \%$ & $74 \%$ \\
\hline $\begin{array}{c}\text { Patients on } 2^{\text {nd }} \text { line RA } \\
\text { biologic treatment, \% }\end{array}$ & $20 \%$ & $22 \%$ \\
\hline $\begin{array}{c}\text { Patients on } 3^{\text {rd }}+\text { line RA } \\
\text { biologic treatment, \% }\end{array}$ & $5 \%$ & $4 \%$ \\
\hline
\end{tabular}

Evaluation of key laboratory measures revealed no significant difference in disease severity between the geographies, per the percentage of patients who tested positive for Rheumatoid Factor, Anti-CCP and HLA-B27 markers. 5EU cohort had significantly higher mean CRP levels and lower mean ESR levels in comparison to the US cohort of patients in remission. Among those with available data, the latest disease assessment scores as measured by standard instruments such as DAS28, VAS and HAQ revealed no difference between the 5EU and US cohorts; Swollen Joint Count and Tender Joint Count were statistically significantly higher for 5EU cohort; Total Sharp Score was also higher among the 5EU cohort, though it did not reach statistical significance. (Table 2)

Table 2: Laboratory and Disease Assessment Measures

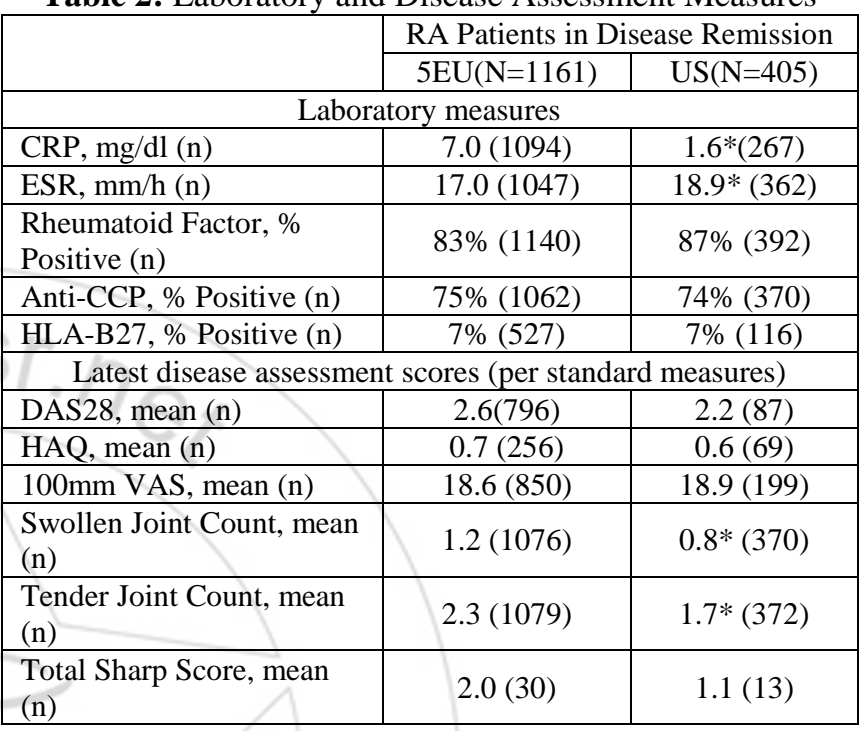

Note: ESR - Erythrocyte sedimentation rate, CRP - Creactive protein, Anti-CCP - anti-cyclic citrullinated peptide antibody, HLA-B27 - Human Leukocyte Antigen B27, DAS28 - Disease Activity Score in 28 joints, HAQ Health Assessment Questionnaire, VAS - 100mm Visual Analog Scale.

*Significantly different at $\mathrm{p}<0.05$

\section{Discussion}

The evolving paradigm of RA patient management involves a multi-dimensional approach encompassing an early diagnosis of the disease and initiation of potent pharmacotherapy, while setting appropriate patientspecific treatment target and applying tight control and relevant therapeutic adaptations to reach this target. EULAR and ACR consistently advocated for clinical remission or low disease activity as key treatment targets [6-8, 10-12]. Treat-to-Target recommendations have historically been non-prescriptive (in terms of pharmacotherapies) and have recommended certain modalities of care delivery and advocated for the general principle of achieving the end target of disease remission or low disease activity [10]. This was the case at the time this study was conducted (in 2011). In this context, the results of this study reporting similar characteristics of RA patients in remission in 5EU and the US, as well as the association of low disease activity consistently observed across these patient cohorts (as measured via validated/standard disease assessment scores) is a significant finding. This depicts the potential influence of EULAR/ACR (in standardizing Treat-to-Target 


\section{International Journal of Science and Research (IJSR) \\ ISSN (Online): 2319-7064}

Index Copernicus Value (2013): 6.14 | Impact Factor (2015): 6.391

guidelines) on physician clinical practices in the respective geographies, benefitting patients.

The mean duration of remission among the US cohort was similar to that of the 5EU averages in this study; however, the duration of remission varied within 5EU depending on the country, with patients in Italy having a lower duration of remission in both $1^{\text {st }}$ and $2^{\text {nd }}$ line treatments. These observed variations may have been influenced by the time since the patient was initiated on the concerned line of therapy (thus, if patients were on a given treatment line for a shorter duration at the time of study data collection, the duration of remission is likely to be shorter).

Although physicians were randomly recruited for this study, the findings may represent only the participating physician practices, and may vary from those of nonparticipating physician practices. This study did not assess the adherence to different facets of Treat-to-Target guidelines in relation to other modalities of RA disease management or care delivery.

\section{Conclusion}

In summary, this is one of the first studies to compare RA patients in remission in the 5EU and US. The characteristics of RA patients in remission in this study were found mostly similar between these geographic clusters, despite the potential variations in healthcare systems and modalities of care delivery. This could be possibly attributed to EULAR/ACR efforts in standardizing the Treat-to-Target guidelines with a focus on clinical remission. There were some variations in duration of disease remission observed within the 5EU, which may warrant scrutiny.

\section{Acknowledgements}

Author would like to acknowledge the contributions from Yao Lu, Richard Hutchings and Amanda Baskett for their research support efforts.

\section{References}

[1] You S., Cho C-S., Lee I., Hood L., Hwang D., et al. A Systems Approach to Rheumatoid Arthritis, PLoS ONE. 2012; 7(12): e51508.

[2] Helmick CG, Felson DT, Lawrence RC, Gabriel S, Hirsch R, et al. Estimates of the prevalence of arthritis and other rheumatic conditions in the United States: part I. Arthritis Rheum. 2008; 58(1):15-25.

[3] Cross M, Smith E, Hoy D, Carmona L, Wolfe F, et al. The global burden of rheumatoid arthritis: estimates from the global burden of disease 2010 study. Ann Rheum Dis. 2014;73(7):1316-22.

[4] Uhlig T, Moe RH, Kvien TK. The burden of disease in rheumatoid arthritis. Pharmaco economics. 2014; 32(9):841-51.

[5] Cutolo M, Kitas GD, van Riel PL. Burden of disease in treated rheumatoid arthritis patients: Going beyond the joint.Semin Arthritis Rheum. 2014; 43(4):479-88.
[6] Smolen J., Landewe R., Breedveld F., Buch M., Burnmester G., et al. EULAR recommendations for the management of rheumatoid arthritis with synthetic and biological disease-modifying antirheumatic drugs: 2013 update. Ann Rheum Dis. 2014; 73(3):492-509.

[7] Sanmartí R, García-Rodríguez S, Álvaro-Gracia JM, Andreu JL, Balsa A, et al. 2014 update of the Consensus Statement of the Spanish Society of Rheumatology on the use of biological therapies in rheumatoid arthritis. Reumatol Clin. 2015; 11(5):27994.

[8] Singh JA, Saag KG, Bridges SL, Akl EA, Bannuru RR, et al. 2015 American College of Rheumatology Guideline for the Treatment of Rheumatoid Arthritis. Arthritis and Care. 2016; 68(1):1-25.

[9] Keyser FD. Choice of biologic therapy for patients with rheumatoid arthritis: The infection perspective. Current Rheumatology Reviews. 2011; 7(1):77-87.

[10] Smolen JS, Aletaha D, Bijlsma JW, Breedveld FC, Boumpas D, et al. Treating rheumatoid arthritis to target: recommendations of an international task force. Ann Rheum Dis. 2010; 69(4): 631-637.

[11] Singh JA, Furst DE, Bharat A, Curtis JR, Kavanaugh AF, et al. 2012 update of the 2008 American College of Rheumatology recommendations for the use of disease-modifying anti-rheumatic drugs and biologic agents in the treatment ofrheumatoid arthritis. Arthritis Care Res (Hoboken). 2012;64(5):625-39.

[12] Smolen JS, Breedveld FC, Burmester GR, Bykerk V, Dougados M, et al. Treating rheumatoid arthritis to target: 2014 update of the recommendations of an international task force. Ann Rheum Dis. 2016; $75(1): 3-15$

\section{Author Profile}

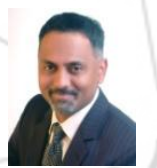

Siva has over 18 yrs of experience in developing product value propositions through market/customer focus, research excellence, and brand strategy support. He is a proven leader and team builder with business and clinical backgrounds. Siva built a strong career in HEOR and Market Access within Pharmaceutical industry through tenures at Human Genome Sciences (GSK), Merck \& Co., Boehringer-Ingelheim and BMS, covering several therapeutic areas and markets. Within healthcare provider sector, Siva has in-depth experience in Long Term Care (LTC) segment, through his tenure at Beverly Enterprises covering the clinical and strategic evaluations across a spectrum of LTC services. In the consulting arena, Siva held a position of Senior Vice President leading Global Treatment Performance Optimization/HEOR Business Unit at TNS (now, part of Kantar) and currently serves as Senior Vice President at Ipsos to lead Global Evidence, Value and Access division/COE, helping clients generate and/or communicate evidence to support their product value propositions. 